\title{
Insight from a COVID-19-Infected Lactating Mother with A Healthy
}

\section{Breastfed Infant}

\section{Yuan Feng1" | Jiaxia Li ${ }^{2 \# ~ \mid ~ J u n c a n g ~ W u ~}{ }^{1}$ | Mingwu Xia ${ }^{1}$ | Wenan Xu${ }^{1 *}$}

\section{*Correspondence: Wenan Xu}

Address: ${ }^{1}$ Department of Neurology, The Affiliated Hefei Hospital of Anhui Medical University, Hefei 230011, Anhui, China; 2Department of Infectious Disease, The Affiliated Hefei Hospital of Anhui Medical University, Hefei 230011, Anhui, China \#Yuan Feng and Jiaxia Li contributed equally to this work

e-mail $\bowtie$ : xuwenan550305@126.com

Received: 18 September 2020; Accepted: 28 September2020

Copyright: (C) 2020 Feng Y. This is an open-access article distributed under the terms of the Creative Commons Attribution License, which permits unrestricted use, distribution, and reproduction in any medium, provided that the original work is properly cited.

\section{ABSTRACT}

During the coronavirus disease 2019 (COVID-19) global epidemic, whether severe acute respiratory syndrome coronavirus 2 (SARS-CoV-2) can be transmitted to infants through breastfeeding has attracted extensive attention. In this report, we present a COVID-19-infected lactating mother with a healthy breastfed infant and discuss related issues. A 33-year-old lactating woman presented with two consecutive positive SARS-CoV-2 tests over 2 days. The test results of her two-month-old daughter and 8-year-old son were both negative. In this patient, the results of IgG and IgM were both positive. Chest CT showed multiple patchy ground-glass shadows in the right lower lung and left lung. She was diagnosed with COVID-19. The patient was advised to suspend breastfeeding. After antiviral treatment with lopinavir/ritonavir and interferon- $\alpha$, the test results for SARS-CoV-2 were all negative on the 4 th, 5 th and 6 th hospital days. A Chest CT reexamination on hospital day 6 suggested remarkable absorption of the shadows in both lungs. There was no recurrence of a positive SARS-CoV-2 test after discharge. The infant's repeated nasopharyngeal swabs for SARSCoV-2 were all negative. Given that close maternal-infant contact is the main way for infants to become infected with SARS-CoV-2, breastfeeding should be suspended to prevent mother-to-infant transmission of SARS-CoV-2.

Keywords: COVID-19, SARS-CoV-2, Infant, Breast-Feeding, Close Contact

\section{Background}

Coronavirus disease 2019 (COVID-19) caused by severe acute respiratory syndrome coronavirus 2 (SARS-CoV-2) shows high potential for person-to-person transmission and thus contributes to a large spread of infection across the globe (Ciotti et al., 2020). Respiratory droplets are the main route of transmission, but SARS-CoV-2 can also be transmitted by close contact or the digestive tract. Family clusters of infection are the major cause of COVID-19 transmission (Hu et al., 2020). During the COVID-19 global epidemic, lactating women, as a special group, have also attracted extensive attention in the context of family clusters of infection, especially regarding whether SARS-CoV-2 could 
be transmitted to infants through breastfeeding (Zhang et al., 2020; Cui et al., 2020). Although maternalinfant transmission through breastfeeding has not been either confirmed or ruled out, limited data on pediatric patients showed that infants could be infected with SARS-CoV-2 via close maternal-infant contact during family life (Le et al., 2020; Wang et al., 2020). Herein, we report a COVID-19-infected lactating woman with a healthy breastfed female infant. However, unlike most reported family clusters where the infants were infected and symptomatic, the infant described here has always been healthy, and repeated nasopharyngeal swabs were negative for SARS-CoV-2.

\section{Case Presentation}

A 33-year-old lactating woman presented with two consecutive positive SARS-CoV-2 tests over 2 days. The patient was initially quarantined due to a neighbor diagnosed with COVID-19 living in the same building. Meanwhile, her father, husband, two-month-old daughter and 8-year-old son were also referred to the quarantine station. The patient was advised to suspend breastfeeding. The next day, the patient developed swelling and pain in the right breast with a transient fever of $38.0{ }^{\circ} \mathrm{C}$. The patient was diagnosed with acute mastitis and received symptomatic treatment. The patient's temperature soon returned to normal. On the 2 nd and 3rd days after quarantine, the family members were tested for SARS-CoV-2 by real-time reverse transcription-polymerase chain reaction (RT-PCR) via nasopharyngeal swabs. The patient, her father and her husband all tested positive in two consecutive tests and was diagnosed with asymptomatic COVID-19, but the test results of her two-month-old daughter and 8-yearold son were both negative. The patient did not have any recent travel history. The possible transmission chain might be through her father, who had been in close contact with the neighbor diagnosed with COVID-19. Then, the patient and her husband were infected by her father, because they were in close contact as part of family life. The female infant and her brother had always appeared healthy. However, the patient had no runny nose, sore throat, cough, expectoration, chest distress or dyspnea, and her baby daughter and older son also did not develop any symptoms.

Physical examination upon admission revealed that vital signs were within normal limits at presentation, with a heart rate of 66 times/min, blood pressure of 110/56 mmHg, respiratory rate of 18 times/min, and temperature of $36.0{ }^{\circ} \mathrm{C}$. Her right breast was slightly swollen, with increased skin temperature and tenderness. There were no abnormal findings in the lungs and heart. Laboratory examinations showed that the routine blood test indicated a white blood cell count of $3.40 \times 10^{* 9 / L}$, a neutrophil count of $1.53 \times 10^{*} \mathrm{~g} / \mathrm{L}$, and a lymphocyte count of $1.57 \times 10^{* 9} / \mathrm{L}$. The $\mathrm{C}$-reactive protein level was $26.91 \mathrm{mg} / \mathrm{L}$, and the serum amyloid A level was $156.1 \mathrm{mg} / \mathrm{L}$. The coagulation test showed that the fibrinogen level was $5.21 \mathrm{~g} / \mathrm{L}$, and the D dimer level was $0.84 \mu \mathrm{g} / \mathrm{mL}$. IgG and IgM were both positive. Chest computed tomography (CT) on hospital day 2 showed multiple patchy ground-glass 
shadows in the right lower lung and left lung (Fig. 1A and Fig. 1B). The patient was diagnosed with COVID-19 and acute mastitis.

The patient received antiviral treatment with lopinavir/ritonavir and interferon- $\alpha$ and symptomatic treatment on the first day of hospitalization. The routine blood test on hospital day 5 showed a white blood cell count of $3.92 \times 10 * 9 / \mathrm{L}$, a neutrophil count of $1.91 \times 10 * 9 / \mathrm{L}$, and a lymphocyte count of $1.62 \times 10^{*}$ / $/$. The C-reactive protein and serum amyloid A levels decreased to $9.84 \mathrm{mg} / \mathrm{L}$ and $89.8 \mathrm{mg} / \mathrm{L}$, respectively. The D dimer returned to normal $(0.34 \mu \mathrm{g} / \mathrm{mL})$. On the 4 th, 5 th and 6th days after hospitalization, the test results for SARS-CoV-2 were all negative by RT-PCR via nasopharyngeal swabs. A Chest CT reexamination on hospital day 6 suggested remarkable absorption of the shadows in both lungs (Fig. 1C and Fig. 1D). The patient was discharged from the hospital on hospital day 7. There was no recurrence of a positive SARS-CoV-2 test during the 14-day quarantine period after discharge. A chest CT reexamination 2 weeks after discharge suggested the complete absorption of the shadows in both lungs (Fig. 1E and Fig. 1F). Her baby daughter and older son were still asymptomatic, and their subsequent repeated nasopharyngeal swabs were still negative for SARS-CoV2.

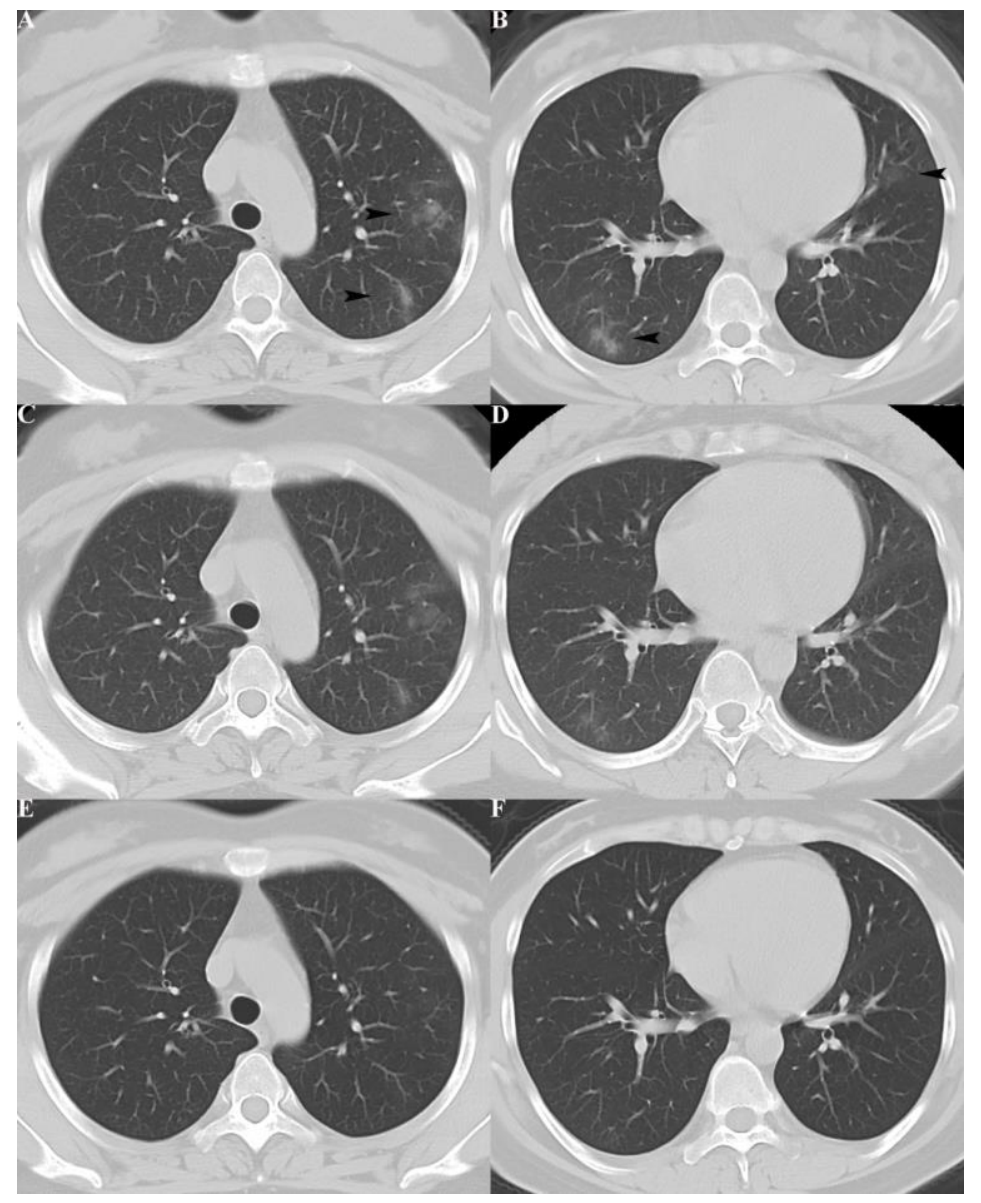

Figure 1: The dynamic evolution of chest CT manifestations in the lactating mother infected with COVID-19 
Chest CT on hospital day 2 showed multiple patchy ground-glass shadows in the right lower lung and left lung (A and B, black arrow).

A Chest CT reexamination on hospital day 6 suggested remarkable absorption of the shadows in both lungs (C and D).

A chest CT reexamination 2 weeks after discharge suggested the complete absorption of the shadows in both lungs ( $\mathrm{E}$ and $\mathrm{F}$ ).

\section{Discussion}

Family clusters of COVID-19 caused by close family contact are the main cause of SARS-CoV-2 infection in infants (Ji et al., 2020), which is undoubtedly the top priority in prevention and control work. In the family cluster of COVID-19 infection described here, all three adults were infected, including the lactating mother, but the 2-month-old infant and her brother were not infected. At present, no SARS-CoV-2 has been detected in the breast milk of mothers with COVID-19 (Zhang et al., 2020; Cui et al., 2020). However, because of the limited number of research cases, these results have not been either confirmed or ruled out. The consecutive negative test results for SARS-CoV-2 in this infant also indirectly indicated that infants might not become infected with COVID-19 through breast milk. However, due to the increasing number of cases during the global outbreak of COVID-19, whether there is SARS-CoV-2 in breast milk needs further study.

Even if infants are not infected through breast milk, close mother-infant contact during family life might also spread the virus to infants. Fortunately, the infant reported here was not infected by SARSCoV-2. It is presumed that the lack of infection in this infant might be related to the presence of asymptomatic patients and/or exposure to a low viral load. The absence of clinical symptoms in all three adults was consistent with this inference. Nevertheless, considering that breastfeeding also carries a risk of contact transmission, breastfeeding should be temporarily suspended until infected mothers' nucleic acid tests turn negative and they are released from medical isolation.

During the COVID-19 global epidemic, limited information is available about children with COVID19, especially infected infants (Cui et al., 2020; Le et al., 2020; Wang et al., 2020; Ji et al., 2020; Yasri and Wiwanitkit, 2020). Infants were often less susceptible to middle east respiratory syndrome coronavirus (MERS-CoV) and severe acute respiratory syndrome coronavirus (SARS-CoV) than adults or had a benign course of infection with mild respiratory symptoms or no symptoms. These results might be related to low exposure, presence of asymptomatic or mildly symptomatic patients, the immature immune system and relatively gentle immune inflammatory response in children and other unidentified 
factors (Memish et al., 2014; Hon et al., 2003). This impression has also been reported for infants during the COVID-19 epidemic. However, these cases are not exactly the same, because people of all ages are susceptible to COVID-19, although the susceptibility may be different (Cui et al., 2020). Therefore, infants who are not infected should also be quarantined for observation and repeated nucleic acid detection, as in our case.

\section{Conclusions}

Whether there is SARS-CoV-2 in breast milk needs further study. However, given that close mother-infant contact is still the main way for infants to become infected with SARS-CoV-2, breastfeeding should be temporarily suspended to protect susceptible infants and prevent mother-toinfant transmission of SARS-CoV-2.

Informed Consent Statement: Informed written consent was obtained from the patient for publication of this report and any accompanying images.

Conflict-of-Interest Statement: The authors declare that they have no conflict of interest.

Author Contributions: Feng Y and Li JX collected the clinical data and drafted the manuscript. Wu JC was a recipient of the obtained funding and reviewed this manuscript. Xia MW and Xu WA reviewed this manuscript.

Acknowledgments: We acknowledge the American Journal Experts (AJE) team for their help in language editing.

\section{References}

Ciotti M, Angeletti S, Minieri M, Giovannetti M, Benvenuto D, Pascarella S, Sagnelli C, Bianchi M, Bernardini S, Ciccozzi M. COVID-19 Outbreak: An Overview. Chemotherapy 2020.

Cui Y, Tian M, Huang D, Wang X, Huang Y, Fan L, Wang L, Chen Y, Liu W, Zhang K, Wu Y, Yang Z, Tao J, Feng J, Liu K, Ye X, Wang R, Zhang X, Zha Y. A 55-Day-Old Female Infant infected with COVID 19: presenting with pneumonia, liver injury, and heart damage. J Infect Dis 2020.

Hon KL, Leung CW, Cheng WT, Chan PK, Chu WC, Kwan YW, Li AM, Fong NC, Ng PC, Chiu MC, Li CK, Tam JS, Fok TF. Clinical presentations and outcome of severe acute respiratory syndrome in children. Lancet 2003; 361: 1701-1703.

Hu Z, Song C, Xu C, Jin G, Chen Y, Xu X, Ma H, Chen W, Lin Y, Zheng Y, Wang J, Hu Z, Yi Y, Shen H. Clinical characteristics of 24 asymptomatic infections with COVID-19 screened among close contacts in Nanjing, China. Sci China Life Sci 2020; 63: 706711.

Ji LN, Chao S, Wang YJ, Li XJ, Mu XD, Lin MG, Jiang RM. Clinical features of pediatric patients with COVID-19: a report of two family cluster cases. World J Pediatr 2020; 16: 1-4. 
Le HT, Nguyen LV, Tran DM, Do HT, Tran HT, Le YT, Phan PH. The first infant case of COVID-19 acquired from a secondary transmission in Vietnam. Lancet Child Adolesc Health 2020; 4: 405-406.

Memish ZA, Al-Tawfiq JA, Assiri A, AlRabiah FA, Al Hajjar S, Albarrak A, Flemban H, Alhakeem RF, Makhdoom HQ. Middle East respiratory syndrome coronavirus disease in children. Pediatr Infect Dis J 2014; 33: 904-906.

Wang D, Ju XL, Xie F, Lu Y, Li FY, Huang HH, Fang XL, Li YJ, Wang JY, Yi B, Yue JX, Wang J, Wang LX, Li B, Wang Y, Qiu BP, Zhou ZY, Li KL, Sun JH, Liu XG, Li GD, Wang YJ, Cao AH, Chen YN. Clinical analysis of 31 cases of 2019 novel coronavirus infection in children from six provinces (autonomous region) of northern China. Zhonghua Er Ke Za Zhi 2020; 58 : E011.

Yasri S and Wiwanitkit V. Clinical features in pediatric COVID-19. Pediatr Pulmonol 2020; 55: 1097.

Zhang YH, Lin DJ, Xiao MF, Wang JC, Wei Y, Lei ZX, Zeng ZQ, Li L, Li HA, Xiang W. 2019 novel coronavirus infection in a three-month-old baby. Zhonghua Er Ke Za Zhi 2020; 58: 182-184. 